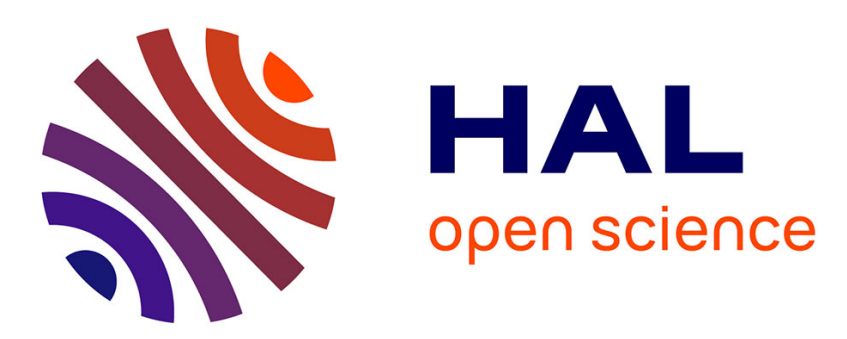

\title{
Contributions de l'espace de concertation à la régulation conjointe. Le cas de Vialeter
}

\author{
Audrey Becuwe, Stéphane Bellini
}

\section{To cite this version:}

Audrey Becuwe, Stéphane Bellini. Contributions de l'espace de concertation à la régulation conjointe. Le cas de Vialeter. @GRH, 2020, n³5 (2), pp.47. 10.3917/grh.202.0047 . hal-02973926

\section{HAL Id: hal-02973926 \\ https://hal.science/hal-02973926}

Submitted on 23 Oct 2020

HAL is a multi-disciplinary open access archive for the deposit and dissemination of scientific research documents, whether they are published or not. The documents may come from teaching and research institutions in France or abroad, or from public or private research centers.
L'archive ouverte pluridisciplinaire HAL, est destinée au dépôt et à la diffusion de documents scientifiques de niveau recherche, publiés ou non, émanant des établissements d'enseignement et de recherche français ou étrangers, des laboratoires publics ou privés. 


\title{
CONTRIBUTIONS DE L'ESPACE DE CONCERTATION A LA REGULATION CONJOINTE.
}

\section{LE CAS DE ViALETER.}

\section{AUdREy BECUWE - STEPHANE BELLINI}

\author{
Revue @grh, n 35 , pages 47 à 72, juillet 2020
}

Résumé : A partir d'une recherche-intervention menée dans une municipalité, nous étudions les effets de l'espace de concertation qui y est initié. Plus spécifiquement, nous cherchons à comprendre en quoi la démarche de concertation est susceptible de favoriser une régulation conjointe (Reynaud, 1997).

Mot-clés : concertation managériale, régulation, changement, recherche-intervention.

\begin{abstract}
The research explains in what a managerial concertation space may favor the joint regulation (TRS-Reynaud, on 1997). From an intervention-research led in a municipality, the TRS allows to understand that managerial concertation may develop the joint regulation.
\end{abstract}

Keywords : managerial concertation spaces, regulation, change management, interventionresearch. 


\section{Introduction}

Enjeu démocratique, la concertation est principalement documentée dans les domaines environnementaux et sociaux autour de différents types de projets. En revanche, la concertation managériale dans les organisations fait l'objet de moins d'attention. Elle permet de mettre en débat les orientations stratégiques de l'entreprise, à la différence des espaces de discussion, qui partent du travail. Des travaux gestionnaires récents (Detchessahar, 2011) ont été initiés sur les espaces de discussion, espaces qui présentent des similarités avec les espaces de concertation dans la mesure où ils sont une instance de régulation du travail (Reynaud, 1979; Detchessar et alii, 2015). Dans la lignée des travaux sur la régulation, notre recherche vise à montrer en quoi un espace de concertation est susceptible de favoriser la régulation conjointe. De cette manière, dans l'intention des initiateurs de la démarche, la concertation permet aux salariés de mieux comprendre les enjeux des orientations stratégiques, et par là même, leur contenu. Comme pour la discussion, la concertation managériale donne ainsi des pistes concrètes au gestionnaire des ressources humaines pour renforcer le sens au travail (Detchessahar et alii, 2015), élément constitutif d'une Qualité de Vie au Travail (QVT). Dans cette perspective, cet article est structuré en quatre parties. Nous ferons d'abord émerger notre problématique de la revue de la littérature car l'examen de la notion de concertation invite à s'interroger sur la capacité de la concertation à concrétiser les régulations conjointes. Nous exposerons ensuite notre méthode de recherche, tendue par une démarche de recherche-intervention, elle-même initiée dans une perspective d'amélioration de la QVT. Puis nous présenterons nos résultats et les propositions de recherche qui émanent de nos travaux.

\section{La concertation, une démarche favorable à la régulation conjointe ?}

La Théorie de la Régulation Sociale (TRS) a inspiré de nombreux travaux étudiant le processus d'appropriation des règles. Si l'espace de discussion est une voie fréquentée de mise en œuvre de la régulation conjointe, d'autres approches sont possibles. La concertation managériale en fait partie. Elle a pour principale caractéristique de mettre au cœur de sa réflexion le débat sur la mise en place d'orientations stratégiques qui, au fil du processus de concertation, en viennent à mettre en discussion le travail. Si les deux notions sont voisines dans leurs intentions de favoriser la parole des acteurs de l'organisation, elles partent de points de départ opposés : la concertation part des orientations stratégiques et en vient à étudier le travail comme un moyen de parvenir à leur mise en œuvre alors que la discussion part d'une mise en débat du travail pour nourrir les échanges sur l'organisation et la stratégie.

\subsection{Dans le sillage de la régulation conjointe}

Avec la TRS, Reynaud (1997) a ouvert un chantier d'études sur le dépassement des impasses et des effets pervers de la régulation de contrôle, vue comme l'usage de règles, d'injonctions et de prescriptions déclinées sous un mode plus un moins normatif et selon des formes variées (notes de services, fiches de poste, règles d'utilisation des outils de gestion...). De nombreux auteurs ont montré la capacité des individus à répondre à l'emprise normative pour reconfigurer des règles de travail (Crozier \& Friedberg, 1977 ; Bernoux, 1981 ; De Terssac, 1992) et à imaginer des régulations autonomes. Reynaud montre que la régulation conjointe permet d'aboutir à un ensemble de règles finalement acceptées de part et d'autre grâce à la mise en débat de la prescription, à une mise à plat des représentations des uns et des autres et à une redéfinition de la prescription, adaptée aux contextes et contraintes du travail (Reynaud, 1997). Pour ce faire, les conversations (Giroux \& Taylor, 1995) - appelées aussi interactions 
discursives (Joffre \& Loilier, 2012) - sont indispensables. La régulation conjointe implique alors "l'élaboration de super-règles, sans supprimer forcément les oppositions entre les sources de régulation, mais en tenant compte des préoccupations et des intérêts de chacun» (Bréchet, 2008, p. 20). Ainsi, la régulation conjointe repose sur «le réalisme et la concession réciproque et sur une part de contrainte »(Reynaud, 1989, p. 371). Au final, la régulation conjointe n'annule ni le contrôle, ni la prescription ; elle est une prescription acceptée car discutée.

La TRS n'est pas exempte de limites et critiques. La TRS est notamment aussi éclairante de phénomènes organisationnels qu'elle est muette sur sa structuration, son pilotage et ses modalités concrètes de mise en œuvre (Paradeise, 2003). Si De Terssac (2003) précise que le processus de régulation comporte plusieurs étapes, il reste néanmoins à spécifier le processus à l'œuvre dans la transition de la régulation de contrôle à la régulation conjointe. L'objectif de notre papier est d'apporter une contribution à la compréhension de ce processus.

Detchessahar (2013) vient combler le point aveugle de la TRS avec la notion d'espace de discussion, pilier opérationnel d'une "ingénierie de la discussion », elle-même vue comme une condition de mise en œuvre des régulations conjointes. L'ingénierie ne désigne pas la construction d'une mécanique infaillible mais montre l'importance d'organiser la discussion. Pour cette raison, l' « espace de discussion » doit être géré, organisé, pensé pour favoriser une discussion voire un débat sur les prescriptions du travail (Detchessahar, 2003, p. 73). Pour Detchessahar (2011), la discussion doit être centrée sur le travail, fréquente, informée sur la base de données factuelles et s'inscrire dans le temps ; elle est encore encadrée et animée par un cadre de et dans l'activité et elle est enfin mémorisée (p. 102). En ce sens, il s'agit de réintroduire les managers sur la scène du travail qu'ils avaient désertée, happés par l'instrumentation de gestion et la participation à des comités transversaux.

\subsection{De la discussion du travail à la conversation stratégique}

Le cœur du projet des espaces de discussion est donc l'organisation de la mise en débat du travail. Mais il est difficile de disjoindre la prescription du travail de son fait générateur, à savoir l'organisation du travail et les orientations stratégiques de l'organisation. A cet égard, Conjard et Journoud (2013) distinguent la discussion opérationnelle de la conversation stratégique, la seconde prenant le relais de la première quand la régulation du travail nécessite des arbitrages à l'échelon organisationnel et stratégique. La conversation stratégique peut alors être "mobilisée au service d'enjeux de régulation ou d'apports de solutions aux problèmes de terrain (de l'opérationnel vers le stratégique) ou de répartition des objectifs (du stratégique vers l'opérationnel) »(Conjard et Journoud, 2013, p. 88).

Pour Detchessahar et alii (2015), trois niveaux d'espaces de discussion sont rassemblés sous le même terme : l'espace de discussion, tel qu'initialement identifié, agit à un niveau opérationnel et vise à réguler les prescriptions du travail entre opérationnels et encadrement de proximité. Les discussions de conception sont réservées aux organisateurs du travail et les discussions stratégiques sont destinées à la direction. L'ensemble forme un chaînage de la discussion, propice à connecter l'espace de discussion opérationnel de la discussion stratégique. C'est en effet en reliant la discussion stratégique à la discussion opérationnelle de manière itérative que le travail peut réellement se transformer. Le chaînage des espaces de discussion peut ainsi permettre une atténuation de la frontière entre conception et exécution. La discussion sur les prescriptions du travail est alors à même de faire évoluer l'organisation du travail voire ses orientations stratégiques. L'articulation entre les différents niveaux de conversation y est vue comme une condition d'évolution des prescriptions du travail, alors que la concertation managériale a pour point de départ les orientations stratégiques. 


\subsection{Les caractéristiques de la concertation managériale}

Il faut d'abord distinguer la concertation managériale du management participatif, ce à quoi nous nous employons dans un premier temps, avant d'en définir les caractéristiques.

Fondamentalement, la concertation et le management participatif se distinguent par leur philosophie managériale. En effet, le management participatif est fondé sur la possible conciliation des logiques économique, politique, au sens d'un rapprochement des logiques d'acteurs aux antagonismes sérieux, et culturelle, aboutissant à un partage de valeurs et à une culture d'entreprise commune (Martin, 1994). Cette philosophie de l'entreprise communautaire n'est pas celle transmise par les démarches de concertation, qui ne se situent pas dans une logique de convergence d'intérêts antagonistes mais au contraire dans une reconnaissance de ces antagonismes pour aboutir, par le dévoilement des intérêts réciproques des acteurs, à des compromis acceptés.

La concertation managériale est inspirée des démarches de concertation publique. La concertation politique et/ou sociétale constitue une logique d'action collective et augmente la coopération des personnes sur un sujet donné dans la mise en œuvre d'une politique (Ornston, Schulze-Cleven, 2015), telle qu'une réforme par exemple. Dans le champ public, elle concerne des objets liés par exemple aux questions environnementales, à l'élaboration et/ou la mise en mise en œuvre des projets urbains (Masboungi, 2002 ; Favreau, 2013) et peut donner lieu à la production d'une charte de concertation (Gardesse, 2011). Elle se caractérise par la mise en œuvre d'un processus de coopération pour la construction et le traitement d'un problème (Beuret et al., 2006) et fait écho au terme anglo-saxon collaborative problem solving (Gray, 1989 ; Anton, 2018). Au fond, il s'agit d'un processus de construction collective de visions, d'objectifs, de projets communs, en vue d'agir ou de décider ensemble. Ce processus repose sur un dialogue coopératif entre plusieurs parties prenantes et vise à construire de nouvelles coordinations autour d'un ou plusieurs objets problématiques (Beuret, 2013). Sa mise en œuvre est questionnée, notamment à propos des zones de flou pouvant apparaître au cours de la démarche. Bratosin (2001) souligne ainsi que la tendance est de confondre la participation au débat avec l'action de décider.

Des travaux étudiant le processus de concertation managériale émergent. Des écrits concernent les structures de santé et étudient les réunions de concertation pluridisciplinaires (Le Divenah et al., 2013). La littérature permet de caractériser la concertation managériale au regard de trois principaux éléments. La littérature aborde d'abord la concertation comme un processus puis définit sa nature et traite enfin des objets de concertation.

Il s'agit tout d'abord d'un processus qui part des orientations stratégiques et prend place dans des « espace-temps » définis. En effet, la concertation s'organise et se planifie en un processus étalé dans le temps (Fortier, 2002), à partir d'une demande prescrite (Schneider, 1987) ou d'une orientation stratégique dont la mise en œuvre est débattue (Regini, 1985). Ce processus prend naissance et perdure dans une relation de confiance qui facilite l'appropriation collective du projet pour les différentes parties prenantes en présence, dans une logique représentative et /ou participative (Thomassian, 2004) et peut s'appuyer sur des outils de gestion, par exemple une matrice de priorisation.

La concertation est ensuite à la fois une activité sociale et une activité de gestion. C'est une activité sociale en ce que la concertation est par nature collective. Elle est un moment de construction d'un objet commun. La concertation combine des moments d'information, de communication et de participation de manière à partager des représentations sur le projet de changement, il s'agit de «dire ensemble » (Bratosin, 2001). C'est aussi une activité de gestion car le partage des représentations permet aux acteurs d'éclairer les décisions en rendant explicites et visibles les points de convergence mais aussi les tensions et contradictions dans la 
construction d'un projet. Il s'agit, pour les acteurs en présence, de chercher une solution allant au-delà de leur propre vision limitée (Gray, 1989). In fine, le processus est traduit en actes managériaux (Cossette, 2004).

Enfin, la concertation exige la définition d'un objet, pivot d'une gestion concertée du changement car une démarche de concertation intervient par nature pour mettre en œuvre un changement. Elle est alors perçue comme un moyen de faire évoluer les préoccupations à l'égard du changement, de préoccupations liées à la raison d'être du changement à celles liées aux modalités de sa mise en œuvre (Rousseau \& Bareil, 2013). Dans ce cadre, un objet favorable à une démarche de concertation doit avoir des implications concrètes (vs discours philosophique), se situer au cœur des préoccupations des directions (vs objet anecdotique), mais aussi être perçu comme légitime et laisser la place à des marges de manœuvre.

Si la concertation managériale part des orientations stratégiques et si ce processus permet in fine l'émergence de représentations collectives à travers les temps d'échange, alors on peut se demander en quoi la concertation peut-elle favoriser une régulation conjointe ? Cette question constitue la problématique du présent article.

La deuxième section de cet article expose le contexte de la recherche et la méthodologie avec laquelle nous avons collecté les données de terrain pour apporter une réponse à cette problématique.

\section{Une recherche au plus près de l'action}

\subsection{Le contexte de la collectivité}

L'étude de la mise en place d'une démarche de concertation porte sur une collectivité territoriale française de 150000 habitants et de 5000 salariés, Vialeter.

\subsubsection{Un environnement de changements}

Le projet est initié peu de temps après un changement de majorité, rompant avec 102 ans d'une gouvernance d'un même courant politique. Ce changement intervient ensuite dans un contexte de baisse des dotations de l'Etat. Cela a eu pour conséquence de réduire fortement le budget de certaines directions, dont celui de la Direction des Parcs et Jardins (DPJ), pour laquelle il s'agit de repenser le fonctionnement et l'activité en tenant compte de cette contrainte. Un projet de direction, initié par un nouveau directeur, intervient dans ce contexte.

D'un point de vue organisationnel, la collectivité est une structure bureaucratique caractérisée par une centralisation des décisions et une hiérarchie bien marquée. A l'arrivée de la nouvelle équipe municipale, le Directeur Général des Services (DGS) a entrepris la restructuration des fonctions supports. Elle s'est accompagnée de la mise en œuvre du management par projet et du pilotage du changement associant démarche de performance locale et amélioration de la qualité de vie au travail.

\subsubsection{La concertation pour accompagner le changement}

Le projet Concertation a été conçu pour développer la concertation managériale dans des projets soumis à une dynamique de changement. Appuyé par le Maire et la DGS, le projet Concertation est animé par un salarié de la collectivité, doctorant et partie-prenante de la recherche, avec le soutien d'une équipe de recherche composée de plusieurs enseignants-chercheurs ${ }^{1}$. Après appel à volontariat, 4 directions, composées de 800 agents ont été incluses dans l'expérimentation. Cet article restitue les analyses relatives à la DPJ, comptant 206 agents parmi lesquels environ 160 jardiniers et une quarantaine d'agents ressortant de fonctions support. Les jardiniers sont

\footnotetext{
${ }^{1}$ Le projet Concertation bénéficie du soutien du Fonds National de Prévention
} 
répartis en 4 secteurs, correspondant chacun à un espace géographique particulier. Un chef de secteur le dirige en s'appuyant sur des chefs d'équipe, chaque secteur étant lui-même divisé en quatre équipes.

Le projet Concertation intervient donc dans un contexte de changement d'orientation politique de la collectivité et de nécessité de trouver des marges de manœuvre financières en interne. La DPJ a été la direction la plus affectée par la diminution des budgets. Ce contexte crée un sentiment de délaissement auprès des agents. Pour faire face aux nouvelles données financières, la direction a lancé un plan de mutualisation de l'utilisation des outils de jardinage entre secteurs. Elle étale également dans le temps le renouvellement du matériel.

\subsection{Une recherche-intervention}

Les termes du contrat de recherche nous liant à l'organisation explicitent notre positionnement : les chercheurs sont appelés en soutien méthodologique à la mise en place de la démarche de concertation. Notre recherche s'inscrit donc clairement dans le champ de la rechercheintervention. A l'échelle de la DPJ, cela se traduit d'abord par une journée de formation à l'exercice d'un style participatif, puis par la conception d'un espace de concertation avec la direction, les chefs de secteur et quelques représentants de fonctions support (bureau d'études interne à la DPJ, DRH, communication, Directeur Général Adjoint-DGA) puis son animation auprès de près de 160 agents de la direction. L'espace de concertation est le temps pensé et organisé pour favoriser la concertation. Il s'est en l'occurrence concrétisé par une journée de mise en débat du projet de direction, sur le temps de travail. Pour que la concertation soit possible pour l'ensemble des salariés, l'opération s'est effectuée en deux temps, soit donc deux journées consacrées totalement à la concertation auxquelles s'ajoute une demi-journée de restitution de l'expression formulée lors de la concertation et de présentation d'un plan d'action qui en résulte.

Les objets de la concertation, le calendrier, la méthodologie ont été progressivement définis en comité de pilotage de manière à impliquer la hiérarchie intermédiaire et surtout à adapter le contenu des échanges aux problématiques du terrain. Les chercheurs-intervenants ont participé à l'architecture de la concertation mais aucun des participants n'en avait une idée précise au moment de s'engager dans la démarche. De plus, la nature collective du travail de conception de l'espace de concertation rend difficile au final l'identification des contributions individuelles.

\subsubsection{Une position d'observation privilégiée}

La nature de l'intervention conduit à connecter étroitement l'objectif pratique (développer une démarche de concertation) et l'objectif de recherche (étudier le déroulement d'une concertation). Cette concordance des objectifs nous situe au plus près des réflexions des acteurs de l'organisation (Hatchuel, 1992 ; Moisdon, 1984). La recherche-intervention n'empêche pas l'habillage rhétorique qui éloigne le discours de la pratique mais elle offre des occasions de le percevoir, de le révéler et d'en faire une donnée. Nous participons ainsi à la construction d'un processus que nous étudions, ce qui est le propre des démarches de recherche-intervention. Nous sommes dans une logique de coproduction des connaissances, «depuis l'intérieur du système et non depuis l'extérieur » (David, 2008, p. 203).

La recherche-intervention étant par nature proche de l'action, son rythme et ses modalités épousent les ruptures de parcours et les méandres plus ou moins turbulents de la vie des organisations. Celle que nous nous efforçons de restituer s'expose d'autant plus à ces aléas qu'elle s'étale sur une période de 30 mois. Le changement de directeur et les contraintes de calendrier ont demandé des adaptations dans le déroulement de la recherche, dont le cheminement relève davantage de la «navigation » que de la «programmation » (Wacheux, 
1996) pour finalement «saisir intelligemment les possibilités d'observation qu'offrent les circonstances »(Girin, 1989, p. 1).

\subsubsection{L'organisation de la réflexivité}

La recherche-intervention nous impose de penser le rapport proximité/distance du terrain tout en assumant notre propre inclusion dans l'objet de recherche (Matheu, 1986). Nous utilisons deux moyens pour cela.

Le recours au collectif est un premier moyen, lui-même décomposé en deux modalités. Les discussions avec l'équipe intervenant au sein d'autres directions de la collectivité, au-delà du binôme affecté à la DPJ, remplit la fonction de "groupe de contrôle »(Girin, 1989), c'est-àdire qu'elle représente un collectif de pairs dont la fonction est de discuter la conduite de la recherche, de la collecte de données et de leur interprétation et faire contrepoids à l'attraction du terrain, redoutable piège des démarches de recherche-intervention. En ce sens le groupe « contrôle » les éventuelles dérives des chercheurs vers des rôles exclusivement d'intervenants, sort les chercheurs de la temporalité et de l'opérationnalité de l'intervention et renforce la logique de production de connaissances inhérente à la recherche. Des réunions régulières des deux chercheurs de l'équipe DPJ constituent une autre occasion de la mise à distance de l'objet de recherche par le collectif et favorisent des temps de réflexivité. Le travail au sein d'un groupe de contrôle et d'une équipe de recherche représente une "triangulation des chercheurs » conduisant plusieurs chercheurs à questionner la collecte et l'interprétation des données (Denzin, 1978). La dialectique proximité/distanciation s'opère autant dans la relation aux acteurs que dans la relation à la problématique.

La rédaction de notes, compilées en un journal de bord, constitue un deuxième moyen d'organiser la réflexivité (Savoie-Zacj, 2000). En plus de conserver la mémoire de faits et impressions, il permet « d'établir un dialogue entre les données et le chercheur à la fois comme observateur et analyste et de permettre au chercheur de se regarder soi-même comme un autre » (Savoie-Zacj, 2000, p. 108).

\subsection{L'abondance du matériau empirique}

Notre matériau est constitué de notes prises lors d'une douzaine de réunions collectives, de multiples réunions avec le directeur des espaces verts et de trois comités de pilotage, de retranscriptions d'entretiens, d'une analyse du premier espace de concertation et de notes d'observations.

\subsubsection{Le recueil des données}

Les données sont constituées de trois éléments, assurant la validité de construit de notre recherche (Hlady-Rispal, 2002) :

- nos notes et retranscriptions d'entretiens, mais tous n'ont pas été retranscrits du fait de refus d'enregistrement de quelques managers intermédiaires ;

- nos notes d'observations des nombreux entretiens préparatoires aux comités de pilotage, comités de pilotage eux-mêmes, et temps d'animation des espaces de concertation ;

- des contributions écrites des agents de la DPJ lors des espaces de discussion, dont nous avons produit une synthèse pour la DPJ dans un document d'une vingtaine de pages.

Les entretiens permettent d'obtenir les représentations de la hiérarchie sur la concertation au moment du démarrage du projet. Nos comptes rendus et notes gardent ensuite des traces des prises de parole et faits et nous autorisent à constater des évolutions dans la relation de la hiérarchie à la concertation. Enfin, l'analyse du premier espace de concertation vise une forme d'objectivation du vécu des jardiniers à l'égard des objets de concertation. 


\subsubsection{Le traitement des données}

Le matériau empirique provient donc de méthodes de collecte complémentaires mais la difficulté de son traitement tient à l'hétérogénéité à la fois de la nature du discours (représentations, avis, vécus, faits, décisions), de son objet (démarche de concertation, travail, orientations de la direction), du mode de collecte, du contexte de sa formulation (en collectif ou en individuel), de leur temporalité (début, milieu ou fin de démarche) et de la fréquence de prise de parole des acteurs (nous nous réunissons souvent avec les chefs de secteur et n'avons eu qu'une occasion d'échanger avec les agents du bureau d'études).

L'intérêt du cadre de l'analyse processuelle s'est progressivement révélé comme étant un cadre d'analyse pertinent pour ordonnancer les données. Nous avons repris nos notes pour en faire ressortir des éléments saillants à partir de la grille de Pettigrew (1990) qui propose une catégorisation des informations en trois thèmes : processus, objet, contexte. Cette grille processus / objet / contexte a donc été le pivot du premier des trois processus d'analyse des données qu'est la description, selon Wolcott (1994). Elle met de l'ordre dans les données dont nous avons évoqué l'abondance. Elle aboutit au processus d'analyse proprement dit, deuxième phase du processus de Wolcott, qui a permis, en interrogeant les données pour leur donner un sens, de mettre en évidence différentes étapes, ce que nous traitons dans la section suivante. Le troisième processus de l'analyse des données est l'interprétation, que nous exposerons dans la discussion. Pour terminer, notre recherche présente les limites inhérentes aux rechercheinterventions et ne permet pas de généraliser nos résultats à d'autres organisations. Des recherches futures dans d'autres contextes sont nécessaires pour confirmer les résultats de la présente étude.

\subsection{Une démarche abductive}

Cette recherche ne procède pas d'une démarche hypothético-déductive, par laquelle nous chercherions à tester un cadre explicatif à travers le terrain. Notre démarche est abductive dans la mesure où nous avons opéré un retour sur les faits après reconstruction d'un cadre explicatif éclairant, à la manière d'une « rétroduction » (Dumez, 2012, p. 3).

En l'occurrence, l'objet de l'intervention était de contribuer à la mise en place d'une démarche de concertation. Notre intention était d'en observer le processus, en nous appuyant sur la grille des stades de préoccupation de Rousseau-Bareil (2013), de manière à identifier l'évolution de l'appropriation de la démarche par les acteurs du terrain. Cependant, notre intention de départ a été contrariée par les difficultés et aléas du terrain : un changement de directeur du service observé puis la relance de la démarche et enfin l'accès à la diversité des réactions de la part des managers de terrain nous ont conduit à choisir un cadre théorique qui permettait de mieux rendre intelligible nos observations, démarche caractéristique de l'abduction (Lorino, 2005). Nous avons donc procédé à "une mise en dialogue des faits empiriques avec des cadres heuristiques de référence » (Anadon \& Guillemette, 2006). Les préoccupations des managers évoluant de manière très variable, il nous est apparu d'abord que la théorie de la régulation sociale apportait un éclairage pertinent à nos observations et ensuite qu'il était plus intéressant de comprendre les moyens de parvenir à une régulation conjointe que de qualifier des stades de préoccupation. Nous présenterons dans la section 4 les propositions auxquelles nous parvenons à l'issue de cette « rétroduction ».

\section{La mise en place d'une démarche de concertation au sein de Vialeter}

La mise en place d'une démarche de concertation ne se résume pas au déroulé rectiligne d'une dynamique planifiée par avance. Elle est le produit d'un entrelacs d'éléments en interaction que nous tentons de démêler pour le rendre intelligible. La « mise en ordre des faits »(David, 2008) 
à laquelle nous nous livrons est un préalable pour ensuite gagner en abstraction et donner une portée théorique à nos propos. L'étude sur 30 mois de la démarche permet d'en discerner différentes étapes. Lors de chacune d'elle, un élément de la grille de Pettigrew (1990) est prégnant. Nous exhumons de notre carnet de bord des notes pour incarner le propos. Au cours des paragraphes suivants, des encadrés mettent en exergue des propos et/ou attitudes des parties-prenantes à la concertation.

\subsection{Première étape : un démarrage laborieux}

Lors des premiers entretiens destinés à préparer la formation, nous relevons une gêne voire une défiance à l'évocation de la concertation. Alors que nous représentons les porteurs de la démarche aux yeux des chefs de secteur, nous sommes confrontés à quelques refus d'enregistrement des entretiens, chose étonnante pour des chercheurs rompus à l'exercice.

Le scepticisme du démarrage de la concertation est justifié explicitement par les chefs de secteur, qui opposent l'affichage de la concertation aux décisions budgétaires non concertées qui ont frappé la DPJ. Au-delà de la réduction du budget, subsistent des incertitudes sur l'année suivante : "on nous demande de faire de la concertation mais pour faire quoi? On ne sait pas quels seront nos moyens l'année prochaine? » dit l'un ; "Il faut faire des efforts, ça, tout le monde le comprend, c'est pas ça le problème mais pour aller dans quelle direction? » dit un deuxième. Les chefs de secteur estiment que le sentiment de mal-être des jardiniers tient essentiellement à un sentiment de relégation et de non-considération. La concertation intervient dans un contexte d'un absentéisme s'élevant à environ $11 \%$ de l'effectif, ce qui a été évalué en interne à 8220 jours de travail par le directeur.

La présence d'intervenants pour mettre en place une démarche non choisie est perçue comme le signe d'une injonction, d'autant plus qu'elle apparaît peu claire pour certains ( $l a$ concertation... euh. Vous pouvez me préciser de quoi il s'agit. Là je ne vois pas très bien... »). Les premiers moments sont donc constitués de mise en question du bienfondé de la démarche, d'autant plus que le directeur de ces premiers moments saisit mal lui aussi comment mettre en œuvre concrètement la démarche, comme l'indique l'encadré 1.

\section{Encadré 1 - Le désarroi du premier directeur}

Alors que nous devions assister à une réunion de service lors de laquelle il devait exposer sa démarche en proposant un objet de concertation et des modalités de travail, le directeur nous apprend $15 \mathrm{mn}$ avant ladite réunion qu'il compte sur nous pour l'animer, lui-même ne sachant pas comment la conduire. Face à sa position réitérée, nous finissons par faire de la réunion un temps d'échange sur le contexte de la DPJ et sur les représentations de la concertation des uns et des autres.

Le changement d'interlocuteur marque une rupture dans le déroulement de la démarche de concertation : le directeur des parcs et jardins est affecté à d'autres missions, laissant un poste vacant pendant 8 mois. Pendant ce temps, la mise en place d'une démarche de concertation est au point mort alors que la collectivité la met en place dans d'autres directions et organise un séminaire des cadres sur le sujet.

\subsection{Deuxième étape : une préparation active de la concertation}

Au sein de la DPJ, un nouveau directeur compte s'appuyer sur la démarche de concertation en s'y engageant activement. Des entretiens en direct ou à distance ont lieu pour préparer une démarche de concertation en lien avec le projet de direction. 
Près de 18 mois après le début de l'intervention et les soubresauts de sa mise en place, un comité de pilotage se réunit pour organiser un espace de concertation. Il s'agit de préparer un temps spécialement dédié à la concertation, organisé et animé pour la favoriser. Il est constitué du directeur, de ses deux adjoints, des chefs de secteur, de la DRH, d'une représentante du service communication et du Directeur Général Adjoint, affecté à cette direction, et des deux chercheurs-intervenants.

Le comité de pilotage se réunit au moment où un projet stratégique se prépare au sein de la DPJ. Il décline en orientations opérationnelles l'objectif général de redéfinition du rôle du jardinier, tenant compte à la fois de l'évolution réglementaire (usage des produits phytosanitaires notamment) et des contraintes budgétaires et humaines. Cela affecte certaines pratiques professionnelles (méthodes de désherbage, fauche tardive, couverture des pieds d'arbre et massifs, choix de plantations tenant compte de leur entretien futur et de l'usage des espaces...).

Le comité de pilotage tient compte de ce projet au moment de définir les objets de concertation. Il s'attache également à traiter des modalités d'organisation y compris les plus précises : nombre de personnes à inviter, durée et lieu de la concertation, période propice à cet exercice, modalités d'animation, répartition des rôles. Le discours initial questionnant le bienfondé de la concertation cède progressivement la place à un discours sur les modalités de sa mise en œuvre.

Certains acteurs s'y engagent avec enthousiasme. C'est le cas du chef du bureau d'études. Il est force de proposition et cherche avec les membres du comité de pilotage à la faire aboutir à des propositions concrètes, comme le souligne l'encadré 2 .

\section{Encadré 2 - Une participation constructive}

Lors du second comité de pilotage, le chef du bureau d'études propose un schéma de concertation pour les futurs projets de création, rénovation ou aménagement des espaces verts. Ce schéma formalise une démarche de co-conception des projets dont l'absence est déplorée par une majorité de jardiniers et fera l'objet d'un travail de concertation à part entière. Il s'agira notamment d'incorporer formellement des temps de concertation dans la réalisation d'un nouveau projet et de préciser le rôle des participants.

\subsection{Troisième étape : le déroulement des espaces de concertation}

Les journées consacrées aux espaces de concertation constituent un temps fort de la démarche. Nous en précisons l'organisation, le rôle des chefs de secteur et la nature de l'expression des jardiniers.

\subsubsection{L'organisation des espaces de concertation}

Pour que l'objet de concertation soit le résultat d'une décision conjointe, les agents de la direction sont d'abord sollicités pour s'exprimer sur leurs activités les plus significatives à leurs yeux. La concertation est organisée pour qu'ils positionnent des étiquettes individuellement sur des panneaux pour traiter ensuite les avis individuels en petits groupes, animés par les chefs de secteur. Après regroupements des avis en catégories, hiérarchisation de leur importance, un thème est retenu pour en faire des objets de concertation plus précis. L'opération se répète à deux reprises pour que l'ensemble du personnel de la DPJ puisse s'exprimer. Les objets de concertation émergent de ces deux journées. Ils ne sont pas identiques puisque l'activité de désherbage est retenue la première journée, en raison du changement de pratiques à promouvoir, et la conception des projets de la DPJ est choisie lors de la seconde, du fait d'un manque de collaboration ressenti entre les agents du bureau d'études et les jardiniers. 
Les objets de concertation, objectivés par le relevé de la fréquence de citations, finissent par avoir un effet structurant. Ils fixent le discours et deviennent le pivot de la concertation. $A$ contrario, d'autres items comme les accidents du travail par exemple ne font l'objet d'aucune discussion alors qu'ils mériteraient d'être débattus selon la DRH. Ils sont dans les faits totalement absents des échanges, évoqués ni par les jardiniers, ni par les chefs de secteur.

\subsubsection{Le rôle des chefs de secteur}

Les chefs de secteur participent au comité de pilotage et sont pour le directeur ses relais et les vecteurs de remontées d'informations du terrain. Ils expriment au départ leur frilosité quant à la démarche de concertation mais contribuent au fil du temps à la construire et acceptent volontiers d'en être des animateurs. Pour autant, tous n'ont pas la même relation aux jardiniers dans leur manière de mener la concertation, où certains écoutent sans intervenir alors que d'autres n'hésitent pas à rappeler les consignes, à signaler des propos hors sujet voire à préciser eux-mêmes certaines propositions émises par les jardiniers. Leurs styles d'animation sont donc différents et peuvent aboutir, avec un fil directeur identique pour tous, à des dynamiques différentes, ce qui se concrétise par des durées d'ateliers variant du simple au double.

\subsubsection{L'expression des jardiniers}

Les jardiniers constituent l'effectif principal de la DPJ. Ils appréhendent très diversement l'idée de concertation, certains y voyant l'espoir d'une écoute quand d'autres le perçoivent comme une agitation stérile dont ils pronostiquent la faiblesse des effets. Durant les espaces de concertation, alors que l'on craignait une retenue excessive du fait du caractère inédit d'une réunion collective, en présence du directeur, du DGA et de l'encadrement intermédiaire, les échanges sont nombreux et directs. Le tout premier échange relève d'une posture d'opposition qui ne parvient cependant pas à s'imposer dans les échanges comme le montre l'encadré 3 .

\section{Encadré 3 - Posture et concertation}

L'organisation de la journée vient d'être présentée. Un jardinier lève la main pour demander la parole, qu'il utilise en s'adressant au DGA pour signaler la diminution des moyens décidée l'année précédente. Le DGA rappelle le contexte et le contenu des discussions passées avec ce jardinier au titre de son mandat syndical mais repositionne la finalité des espaces de concertation. La question suivante embraye sur un sujet tout à fait opérationnel. Le DGA se met en retrait et le sujet ne reviendra plus dans les échanges.

Cependant, la concertation s'engage sur des niveaux d'informations inégaux, créant des incompréhensions. L'exemple de l'incompréhension au sujet de l'achat de matériel est ainsi flagrant, comme le relate l'encadré 4 .

\section{Encadré 4 - Incompréhension sur les marchés publics}

Dans un sous-groupe composé de jardiniers, la discussion porte sur l'achat de matériel. Un jardinier déplore qu'on ne puisse acheter le matériel souhaité, qu'il voit pourtant dans les magasins de bricolage. Le chef de secteur, animateur de la séance estime alors qu' "il faut une petite explication » sur les marchés publics avant de poursuivre la concertation. Il rappelle les règles d'achat en collectivité. A la suite de son explication, le jardinier revient à la charge : "mais le directeur, lui, il pourrait pas acheter le matériel ? ». Le chef de secteur doit préciser que le directeur doit lui aussi s'y soumettre et que cette règle ne vaut pas uniquement pour cette collectivité mais qu'elle est nationale. "Mais le Maire, lui, il pourrait pas décider d'acheter quand même?». Le chef de secteur me regarde en soupirant. Je prends le relais en indiquant que cette règle est conçue pour éviter les dérives de corruption. 
D'une part, la démarche de concertation permet de recueillir ce type de représentations par l'expression qu'elle favorise, d'autre part, elle peut contribuer à résorber les écarts de connaissances mais de tels écarts représentent également une limite de la concertation.

\subsection{Quatrième étape : la fragile mise en place du plan d'actions}

Les deux espaces de concertation ont lieu en décembre et janvier. Il est convenu d'en tirer les enseignements rapidement et d'enclencher un plan d'actions de manière à profiter de la dynamique tout en envoyant aux jardiniers le signal d'une écoute de leur parole. Pour des raisons d'agenda, de problème de diffusion de notre synthèse en interne, le comité de pilotage consacré au bilan se réunit deux mois plus tard et dresse des pistes qui restent à approfondir. Le directeur éprouve le besoin d'un $2^{\mathrm{e}}$ comité de pilotage pour aboutir à des propositions finalisées. Ce qui devait être une grande réunion de restitution avec l'ensemble des agents de la DPJ se transforme donc en comité de pilotage, ce que nous apprenons la semaine précédente. Nous arrivons fin mai. L'activité de la DPJ bat son plein et les premiers départs en vacances se profilent. La réunion de restitution n'aura finalement lieu qu'en septembre. Huit mois se seront donc passés entre le dernier espace de concertation et la réunion qui en fait le bilan. La dernière journée plénière dédiée à la concertation met en exergue une évolution du discours des agents, explicitée en encadré 5.

\section{Encadré 5 - Les trois objets du discours des agents}

Lors de la dernière journée plénière, rassemblant l'essentiel des personnels de la direction, aucune posture d'opposition n'est recensée. Les questions et remarques sont de trois ordres. Tout d'abord, de nombreuses questions relatives aux choix de la direction sont posées, notamment sur l'évolution du périmètre d'activité de la DPJ et sur les mutualisations avec d'autres services. Apparaissent également des propositions concrètes au cours de la séance, sur l'utilisation du compte Facebook de la direction pour sensibiliser à la propreté, sur l'opportunité d'installer des poubelles de tri sélectif dans les parcs, sur l'expérimentation du désherbage par le vinaigre... Enfin, d'autres questions ou remarques sont relatives au travail des agents. A cet égard, l'activité de propreté est souvent citée pour exprimer le sentiment de manque de respect des parcs et jardins par le public.

Le contenu des espaces de concertation fait émerger des propositions concrètes. En soi, c'est un signe encourageant pour la suite de la démarche. Cependant, d'autres signes invitent à tempérer ce pronostic car l'ensemble de l'encadrement n'est pas convaincu par la démarche, comme le montre l'encadré 6 , or sa pérennisation dépendra de la mise en œuvre sur le terrain des propositions formulées au cours des espaces de concertation.

\section{Encadré 6 - L'ambivalence des positions}

Le comité de pilotage de clôture est l'occasion d'un bilan. Un chef de secteur manifeste son intérêt pour la démarche, insistant sur le fait " qu'on devrait organiser des temps de concertation plus souvent ». Un autre signale que "cela a répondu à une demande mais, maintenant, il faut que ça suive ». Un troisième est plus partagé : "la concertation, c'est bien mais ça prend du temps. Pendant ce temps, le travail n'avance pas. Avec la charge de travail que j'ai sur le secteur, je ne pourrai pas retirer les agents du travail pour se concerter. A un moment, le travail doit se faire ».

De son côté, l'adjoint au directeur est davantage centré sur l'expertise et le contrôle de gestion. Sous le mandat du premier directeur, il a effectué un imposant travail de recensement et 
d'identification du niveau de mutualisation des outils de travail. Ce travail est peu repris par le nouveau directeur, qui y voit un document «très technique » et " qui oublie l'aspect humain ». Alors qu'un jardinier questionne le directeur adjoint sur l'organisation de la journée, ce dernier indique, geste à l'appui, qu'il n'est pour rien dans l'organisation et qu'il ne connaît rien du déroulement de la journée. De fait, il était en congés lors du premier comité de pilotage mais la tonalité de sa remarque étonne. On peut y voir l'expression d'un doute quant à la pertinence de la démarche, ce qu'une discussion en aparté confirmera, restituée dans l'encadré 7.

\section{Encadré 7 - La concertation vue comme un espace de légitimation de la plainte}

En aparté lors du second espace de concertation, l'adjoint au directeur confie voir la concertation comme une légitimation de la plainte et/ou de la contestation. Par exemple, alors que beaucoup de jardiniers estiment que le matériel fait défaut, n'est pas bien choisi et qu'il est mal entretenu, le directeur adjoint ne partage pas ce constat. Pour lui, les jardiniers se dédouanent de leurs propres responsabilités dans l'entretien du matériel. Il indique également que certains matériels ont été achetés à la demande de jardiniers et sont en fait très peu utilisés.

\section{Le cheminement vers une régulation conjointe}

La problématique de cette recherche s'énonce dans les termes suivants : en quoi la concertation peut-elle favoriser une régulation conjointe dans le cadre d'une démarche de changement ? Dans le cas présenté, les orientations stratégiques de la municipalité renforcent les contraintes de la DPJ qui propose un projet stratégique redéfinissant les rôles des jardiniers. La démarche de concertation mise en place vise à tendre vers une régulation conjointe car elle cherche à organiser des retours des jardiniers par rapport au projet stratégique. Ce faisant, il ne s'agit pas seulement d'énoncer des prescriptions normatives qu'il faudra faire appliquer mais d'ajuster les orientations en combinant les contraintes financières et matérielles, les projets du directeur et le vécu des acteurs de terrain. La mise en débat de l'orientation stratégique s'inscrit dans la TRS (Reynaud, 1989) mais la démarche employée au sein de la DPJ se différencie de la démarche des espaces de discussion dans la mesure où l'objet mis en débat n'est pas le travail (Detchessahar et alii, 2015) mais l'orientation stratégique.

\subsection{L'espace de concertation : un moyen d'organiser une régulation conjointe}

Lors de la première étape, faute d'organiser les espaces dédiés, la concertation apparaît davantage comme une forme de régulation de contrôle dont nos interlocuteurs cherchent à se distancer. Elle est vécue comme une injonction décalée du terrain et en réaction, la hiérarchie intermédiaire en questionne la pertinence, montrant sa capacité à mettre en cause la régulation de contrôle (Reynaud, 1997).

Puis au cours de la $2^{\mathrm{e}}$ étape, l'espace de concertation s'organise en un dispositif formalisé. Le processus est pensé dans ses détails, comme la composition du comité de pilotage, la manière de solliciter la parole, la taille des groupes de travail et les dates des espaces de concertation. Ces premiers éléments montrent que l'organisation de la démarche est une condition pour tendre vers des régulations conjointes (Paradeise, 2003 ; De Terssac, 2003). Il n’y a donc pas de concertation dans l'absolu mais des démarches de concertation données dont le cas de la DPJ donne un exemple de design possible en s'attachant à détailler le processus et les objets de concertation.

La concertation s'organise encore à travers la définition des objets dont certains sont propices à la concertation. Le schéma de concertation proposé pour les projets d'envergure peut devenir un outil de la régulation en ce qu'il permet d'infléchir les orientations stratégiques au regard 
des possibilités du travail. Si l'espace de concertation porte sur la mise en œuvre des orientations stratégiques et non pas sur leur définition, en réalité, celles-ci pourraient être amendées via la prise en compte des possibilités réelles du travail.

La $3^{\mathrm{e}}$ étape laisse voir également une régulation conjointe s'opérer sur la base de la préparation présentée préalablement : en définissant conjointement des objets de concertation, en impliquant les agents dans la démarche, des représentations s'exposent et se confrontent, comme lors de l'échange sur la règle des marchés publics exposé dans l'encadré 4, pour finalement se rapprocher. Des propositions sont formulées, dont on peut penser qu'elles constituent un élément favorable de leur acceptation ultérieure.

L'organisation de l'espace de concertation nécessite de combiner avec les contraintes de l'opérationnel. C'est ce que la 4 étape montre. Ainsi, pour des questions de calendrier et de cumul de reports de dates, on assiste à un décalage de 8 mois entre le $2^{\mathrm{e}}$ espace de concertation et le bilan. De la même manière que la discussion nécessite une ingénierie (Detchessahar, 2011), la concertation nécessite d'être réfléchie dans ses détails organisationnels pour qu'elle favorise l'exercice de la parole et soit crédible. Le souci de ces détails dépasse largement la seule considération logistique mais produit du sens; il n'est pas accessoire mais essentiel. L'éloignement des dates de concertation, s'il est compréhensible sur le plan du fonctionnement de la DPJ, fait ainsi perdre du crédit à la dynamique créée.

Enfin, cette organisation sur la forme doit être compatible avec une liberté de parole sur le fond et doit permettre de mettre en débat les orientations stratégiques. Cela suppose pour les managers d'accepter de ne pas maîtriser totalement ce qui est habituellement de leur prérogative.

En réponse à notre problématique, le cas de la DPJ de la Mairie de Vialeter montre que la concertation managériale peut être le support d'une régulation conjointe si elle repose sur un dispositif formel, adapté aux contraintes temporelles des opérationnels et centré sur des objets mobilisateurs.

\subsection{L'espace de concertation, un artefact au service de la régulation conjointe}

Alors que la littérature sur la concertation comme sur les espaces de discussion met l'accent sur l'ingénierie des dispositifs de soutien, nos observations montrent qu'elle ne saurait suffire à créer et à maintenir une dynamique favorable à la concertation. Le dispositif de soutien est à considérer comme un artefact baptisé espace de concertation et produisant des effets sociaux. Il intervient tout d'abord dans des jeux sociopolitiques dans lesquels les acteurs font valoir un point de vue sur le travail, parfois issu d'une position de représentation du personnel comme le signale l'interpellation d'un agent dans l'encadré 3, mais aussi des intérêts et questionnements plus concrets, ce qu'indiquent les objets du discours présentés dans l'encadré 5 . En ce sens, l'espace de concertation a demandé un dévoilement des préoccupations et intérêts des acteurs. Pour Bréchet (2008), cela constitue précisément une condition de la régulation conjointe. Le dispositif instrumental est également accepté s'il produit du sens, faute de quoi les acteurs le perçoivent comme une prescription décalée des contraintes du terrain, contraignant l'action plus que la facilitant. Le premier directeur la percevait comme telle. Après plusieurs mois et un changement de directeur, la production de sens se manifeste à travers l'engagement des acteurs en vue de mettre en œuvre un plan d'action opérationnel; ils y voient une solution pratique à des problèmes d'organisation, ce qu'illustre la proposition d'un schéma de concertation. Les comités de pilotage ont permis cette construction de sens. Cependant, la production de sens n'opère pas de manière mécanique car avec le même dispositif, le chef de secteur cité dans l'encadré 6 tend à qualifier la concertation comme une aimable conversation mais peu productive tandis que le directeur adjoint y voit une légitimation de la contestation (encadré 7). Cette situation amène à considérer l'espace de concertation non pas pour ses caractéristiques 
propres (taille des groupes, fréquence des réunions, nature de l'objet de la concertation) qui agiraient indépendamment de toute appropriation mais pour les effets qu'ils produisent dans un contexte relationnel donné, ces effets étant en permanente évolution. Nous rejoignons Grimand (2012), pour qui les outils de gestion et les dispositifs formels de management doivent être observés à travers l'appropriation qu'ils suscitent et pas seulement pour leur seul design.

En réponse à notre problématique, le cas de la DPJ de la Mairie de Vialeter montre que la concertation managériale peut être le support d'une régulation conjointe si l'espace de concertation est pensé dans son statut d'artefact, permettant le dévoilement des intérêts des acteurs par les interactions discursives qu'il provoque (Joffre \& Loilier, 2012) et favorisant une production de sens. De ce fait, le statut de l'espace de concertation est à penser dans ses dimensions symbolique et politique, au-delà de sa dimension objective.

\subsection{Les managers, des agents de la régulation conjointe}

Nous relevons que l'espace de concertation ne devient pas lui-même un pivot de la régulation conjointe sans un agent de la régulation. Nous qualifions les managers, ici les chefs de secteur, d'agents de la régulation car ils animent les espaces en favorisant plus ou moins le dévoilement des intérêts, l'expression sur la compatibilité des orientations avec les contraintes du terrain, constitutifs de la régulation conjointe. Les temps d'échange et les modalités d'interaction très variables entre les groupes de travail montrent la variété des styles d'animation. Dans le cadre des espaces de concertation, les managers ne sont pas des rouages passifs de l'organisation et des dispositifs formels, se coulant automatiquement dans le moule conçu pour eux. C'est en cela que nous évoquons la notion d'agent de la régulation, nécessaires à l'animation de ces dispositifs formels et à la construction de sens.

En tant que chercheurs-intervenants, nous avons-nous-même joué ce rôle d'agent de la régulation conjointe, en organisant les espaces de concertation de telle sorte que la parole puisse s'exprimer. Ce rôle d'agent de la régulation peut aussi conduire à distribuer l'information nécessaire au dialogue, à la manière de ce que restitue l'encadré 4 sur l'incompréhension sur les marchés publics. Le manager de proximité est bien plus qu'une courroie de transmission neutre et impassible aux retours du terrain. En cela, nous rejoignons Detchessahar (2013) qui revendique sa présence pour l'organisation du dialogue. Nos travaux montrent que pour cela, il semble nécessaire de commencer par manager les managers, c'est-à-dire par les convaincre en premier lieu de l'intérêt du changement entrepris. La surveillance de l'évolution de leurs préoccupations est un indice de leur capacité à eux-mêmes devenir moteurs des changements (Rousseau \& Bareil, 2013). Le rôle du manager de proximité est de faire le lien entre la concertation en des temps identifiés et le management au quotidien.

Un des risques de la concertation n'est pas seulement d'être empêchée par l'absence d'espaces dédiés, comme Detchessahar (2011) le souligne à propos de la discussion, mais d'un défaut d'appropriation par ses relais censés être des agents de la régulation conjointe. De la même manière que Detchessahar (2013) identifie des profils de managers en situation de management empêché par l'envahissement du reporting (le manager héroïque, le manager résigné et le manager complice), l'étude des profils des agents de la régulation conjointe mériterait d'être approfondie.

En réponse à notre problématique, le cas de la DPJ de la Mairie de Vialeter montre que la concertation managériale peut être le support d'une régulation conjointe si des agents de la régulation, notamment les managers, adoptent un rôle qui lui est favorable. Plus précisément, il s'agit pour eux d'encourager la parole et de diffuser les informations nécessaires à la concertation. 


\subsection{Une régulation conjointe qui rend indissociable la concertation sur les orientations stratégiques et la discussion sur le travail}

Si l'espace de concertation se distingue de l'espace de discussion du fait d'objets centraux différents, il est difficile d'envisager l'espace de concertation indépendamment de tout espace de discussion, les orientations stratégiques se répercutant in fine sur le travail des agents de terrain. Le dernier espace de concertation montre que les questions et remarques des jardiniers portent à la fois sur le travail et la stratégie de la direction. Ainsi, l'orientation vers un désherbage sans recours aux produits phytosanitaires modifie les pratiques professionnelles. La concertation ouvre la voie à des échanges sur la manière de mettre en œuvre l'orientation stratégique qui elle-même inspire la prescription et la rapproche de ce fait de la discussion. Avec un point de départ inversé, c'est-à-dire la discussion sur le travail, Detchessahar et alii (2015) parviennent au même constat de la nécessité d'un chaînage entre les espaces.

Il ne s'agit pas de mettre en compétition ou en opposition les deux méthodes d'organisation de l'expression collective mais de signaler l'inséparabilité fondamentales entre la discussion sur le travail et la concertation sur les objectifs stratégiques. La disjonction de l'un et de l'autre n'a pas de sens et procéderait d'un aveuglement. Orientation stratégique et travail sont intimement liés et n'agissent pas en deux blocs étanches l'un de l'autre. Cela signifie que la régulation conjointe opère quand la concertation managériale sur la mise en œuvre des orientations stratégiques s'articule à une discussion sur le travail (Conjard \& Journoud, 2013).

\section{Conclusion}

A l'issue de notre intervention sur les 30 mois, une démarche concertation a été mise en place au sein de la direction étudiée et répond ainsi aux objectifs opérationnels initiaux. L'émergence du schéma de concertation - schéma qui n'existait pas et qui n'était pas prévu au début de la démarche - est le témoin de l'institutionnalisation de la démarche au niveau de la direction. Cette expérimentation, novatrice à l'échelle des organisations françaises, tend plus globalement à s'institutionnaliser au sein de la mairie par la diffusion du dispositif vers les autres directions.

Les résultats de notre recherche-intervention aboutissent à quatre propositions explicatives qui sont autant de contributions de la concertation à la régulation conjointe :

- D'abord, pour aboutir à une régulation conjointe, la concertation s'organise en un dispositif formel, à travers la définition d'objets adaptés, et doit se combiner avec les contraintes temporelles de l'opérationnel. Elle ne s'improvise pas ;

- Ensuite, l'espace de concertation peut être un moyen de parvenir à une régulation s'il permet le dévoilement des représentations et intérêts des acteurs par les interactions discursives qu'il provoque (Joffre \& Loilier, 2012) et une production de sens. En ce sens, l'espace de concertation est à considérer davantage comme un artefact que comme un dispositif aux caractéristiques agissant indépendamment de l'appropriation des acteurs.

- De plus, les agents de la régulation peuvent favoriser les interactions, en organisant les échanges, en stimulant l'expression et en diffusant l'information nécessaire à la concertation. Ce sont notamment les encadrants de proximité. Ce faisant, ils contribuent fortement à faire de la concertation un support d'une régulation conjointe.

- Enfin, pour aboutir à une régulation conjointe, la concertation sur la mise en œuvre des orientations stratégiques doit s'articuler à une discussion sur le travail.

L'apport théorique de notre recherche réside dans l'exploration de nouvelles modalités de la mise en œuvre de la régulation conjointe, modalités peu explicitées par Reynaud (Paradeise, 2003), en partant d'une concertation sur les orientations stratégiques d'une organisation. 
Notre recherche met également en exergue un second impensé de la TRS, à savoir l'importance fondamentale d'agents de la régulation, moteurs dans la démarche. Il est alors nécessaire de développer les compétences des managers en matière d'animation mais aussi de création et de recréation des règles (Desmarais \& Abord de Chatillon, 2010), et enfin de les accompagner dans leur rôle de traduction. Plus généralement, il s'agit pour les managers de trouver un équilibre entre les activités de reporting et de contrôle et celles relatives à la régulation.

D'un point de vue managérial, nos travaux invitent à penser dans le détail la démarche de concertation, sur des aspects aussi concrets que le calendrier, les lieux de réunion, les méthodes visant à favoriser l'expression du plus grand nombre, la mobilisation la plus large possible. De plus, l'objet concret de la concertation est central dans ce type de démarche : il s'agit de définir un objet porteur de sens et pour lequel les salariés peuvent apporter leur expertise, issue de la connaissance du terrain. Pour cela, il est nécessaire que les modalités de mise en œuvre soient ouvertes. Nos propositions suggèrent également de sensibiliser voire de former les encadrants de proximité à des méthodes d'animation susceptibles de favoriser les interactions (post it, chapeaux de Bono, photo-langage ou autres méthodes de résolution de problème participatives). Enfin, il s'agit de préparer les managers à voir évoluer la concertation sur la stratégie vers une discussion sur le travail, les deux étant intimement liés. Le schéma de concertation permet d'articuler concertation et discussion.

Ces résultats invitent à prolonger la recherche d'une part en testant les propositions formulées dans cet article et d'autre part en explorant davantage la manière avec laquelle les managers deviennent eux-mêmes des agents de la régulation.

\section{Références}

ANADON M., GUILLEMETTE F. (2006), « La recherche qualitative est-elle nécessairement inductive », Actes du colloque de l'Association pour la recherche qualitative, ACFAS, Montréal.

ANTON P. (2018), "Manager des espaces de concertation dans le cadre d'un projet de transformation au sein d'une collectivité locale : quels liens avec la satisfaction au travail, le changement et les comportements pro-sociaux ? », Question(s) de management, 2018/2, n²1, p. 55-64.

BERNOUX P. (1981), Un travail à soi, Privat.

BEURET, J.-E., PENNANGUER S., TARTARIN F. (2006), «D'une scène à l'autre, la concertation comme itinéraire », Natures, Sciences, Sociétés, 14 (1).

BEURET J.-E. (2013), «Concertation (démarche de)», in CASILLO I., BARBIER R., BLONDIAUX L., CHATEAURAYNAUD F., FOURNIAU J.M., LEFEBVRE R., NEVEU C., SALLES D., Dictionnaire critique et interdisciplinaire de la participation, Paris, GIS Démocratie et Participation.

BRATOSIN S. (2001), La concertation : forme symbolique de l'action collective, L'Harmattan, collection Logiques sociales.

BRÉCHET J.-P. (2008), « Le regard de la théorie de la régulation sociale de Jean-Daniel Reynaud », Revue Française de Gestion, n 184, p. 13-34.

CONJARD P., JOURNOUD S. (2013), «Ouvrir des espaces de discussion pour manager le travail », Management \& Avenir, 2013/5, $\mathrm{N}^{\circ}$ 63, p. 81-97.

COSSETTE P. (2004), L'Organisation : Une perspective cognitiviste, Collection Sciences de l'Administration, Québec, Presses de l'Université Laval. 
CROZIER M., FRIEDBERG E. (1977), L'acteur et le système, Editions du Seuil, Paris.

DAVID A. (2008), «La recherche-intervention, cadre général pour la recherche en management ? », in, DAVID A., HATCHUEL A. et LAUFER R., Les nouvelles fondations des sciences de gestion. Eléments d'épistémologie de la recherche en management, Vuibert, collection Fnege, $2^{\mathrm{e}}$ édition, pp 193-213.

DENZIN.K. (1978), The research act: A theoretical introduction to sociological methods, New York, McGraw-Hill.

DESMARAIS C., ABORD DE CHATILLON E. (2010), « Le rôle de traduction du manager. Entre allégeance et résistance », Revue Française de Gestion, n²05.

DE TERSSAC G. (2003), La théorie de la régulation sociale de Jean-Daniel Reynaud, Débats et prolongements, La Découverte.

DE TERSSAC G. (1992), Autonomie dans le travail, PUF.

DETCHESSAHAR M., GENTIL, S., GREVIN, A., STIMEC, A. (2015), « Quels modes d'intervention pour soutenir la discussion sur le travail dans les organisations ? », @ GRH, (3), 63-89.

DETCHESSAHAR M. (2013), «Faire face aux risques psycho-sociaux : quelques éléments d'un management par la discussion », Négociations, $\mathrm{n}^{\circ} 19$, p. 57-80.

DETCHESSAHAR M. (2011), «Santé au travail. Quand le management n'est pas le problème, mais la solution... », Revue Française de Gestion, n²14/5, pp. 89-105.

DETCHESSAHAR M. (2003), "L'avènement de l'entreprise communicationnelle », Revue française de gestion, $\mathrm{n}^{\circ} 142$, p. 65-84.

DUMEZ H. (2012), «Qu'est-ce que 1'abduction et en quoi peut-elle avoir un rapport avec la recherche qualitative », le Libellio d'Aegis, vol. 8, n³, automne, pp. 3-9.

FAVREAU F. (2013), «Fonder la pratique de la concertation sur la théorie des conventions ? Etude d'un processus de décision participatif au Venezuela », AIMS.

FORTIER J. (2002), «Proposition d'un cadre de référence en concertation». Mémoire de maîtrise. Département des Sciences du loisir et de la communication sociale. Université du Québec à Trois-Rivières.

GARDESSE C. (2011), «La "concertation" citoyenne dans le projet de réaménagement du quartier des Halles de Paris (2002-2010) : les formes de la démocratisation de l'action publique en urbanisme et ses obstacles », Architecture, aménagement de l'espace, Université Paris-Est.

GIRIN J. (1989), «L'opportunisme méthodique dans les recherches sur la gestion sur les organisations », Journée d'étude sur la recherche-action en action et en questions, Ecole Centrale, 10 mars.

GIROUX N., TAYLOR J. (1995), « Le changement par la conversation stratégique », in NOEL A. (Eds), Perspectives en Management stratégique, tome 3, Paris, Economica.

GRAY B. (1989), Collaborating : Finding Common Ground for Multiparty Problems, JosseyBass : San Francisco.

GRIMAND A. (2012), «L'appropriation des outils de gestion et ses effets sur les dynamiques organisationnelles : le cas du déploiement d'un référentiel des emplois et des compétences », Management \& avenir, $\mathrm{n}^{\circ} 54$, juin, 237-257.

HATCHUEL A. (1992), «L'intervention des chercheurs en entreprise », Education Permanente, Numéro 113, pp. 73-88. 
HLADY-RISPAL M. (2002), La méthode des cas. Application à la recherche en gestion. Bruxelles. DeBoeck.

JOFFRE C., LOILIER T. (2012), «L'adaptation vue comme un processus de régulation. Le rôle et la dynamique de la règle dans une organisation du secteur social et médico-social français », Management International, vol. 16, $\mathrm{n}^{\circ} 2$, hiver, p. 39-55.

LE DIVENAH A., DAVID S., BERTRAND D., CHATEL T., VIALLARD M.-L. (2013), «Réunion de concertation pluridisciplinaire : comment décider d'une chimiothérapie à visée palliative ? », Santé Publique 2013/2 (Vol. 25), p. 129-135.

LORINO P. (2005), Contrôle de gestion et mise en intrigue de l'action collective, Revue Française de Gestion, ${ }^{\circ} 159$, pp. 189-211.

MARTIN D. (1994), Démocratie industrielle. La participation directe dans les entreprises, Paris, PUF.

MASBOUNGI A. (2002), Projets urbains en France, Paris, Editions du Moniteur.

MATHEU M. (1986), «La familiarité distante. Quel regard poser sur la gestion de notre société ? », Annales des Mines, Gérer et comprendre, n², mars, pp. 81-95.

MOISDON J.-C. (1984), «Recherche en gestion et intervention », Revue Française de Gestion, septembre, p. 61-73.

ORNSTON D., SCHULZE-CLEVEN T. (2015), Concertation and Coordination, Two Logics of Collective Action, Comparative Political Studies, 48(5).

PARADEISE C. (2003), "La théorie de la régulation sociale à l'épreuve de la pratique », in DE TERSSAC G., La théorie de la régulation sociale de Jean-Daniel Reynaud. Débats et prolongements, éditions La Découverte, coll. «Recherches », Paris, pp. 41-49.

PETTIGREW A.M. (1990), «Longitidunal Field Research on Change: Theory and Practice », Organization Science, vol.1, n 3 .

REGINI M. (1985), « The Conditions for Political Exchange: How Concertation Emerged and Collapsed in Italy and Great Britain », In J.H. GOLDTHORPE (ed), Order and Conflict in Contemporary Capitalism, Oxford, Clarendon Press, pp. 124-142.

REYNAUD J.D. (1989 / 1997), Les règles du jeu - L'action collective et la régulation sociale, Armand Colin, Paris.

REYNAUD J.-D. (1979), « Conflit et régulation sociale. Esquisse d'une théorie de la régulation conjointe », Revue Française de Sociologie, Vol. 20, 367-376.

ROUSSEAU C., BAREIL C. (2013), «Evolution de l'appropriation et des préoccupations des cadres intermédiaires en contexte de changements simultanés », Question(s) de management $\mathrm{n}^{\circ} 3, \mathrm{p} .81-100$.

SAVOIE-ZAJC L. (2000), « La recherche qualitative/interprétative », dans KARSENTI T., SAVOIE-ZAJC L., Introduction à la recherche en éducation, Sherbrooke : CRP.

SCHNEIDER R. (1987), Gestion par concertation, Montréal, Agence d'Arc.

THOMASSIAN M. (2004), « La concertation du public au niveau local : du monologique au dialogique. Le cas des projets de transport », Management International, vol. $9, \mathrm{n}^{\circ} 1$, novembre, p. 99-116.

WACHEUX F. (1996), Méthodes qualitatives et recherches en gestion, Economica. 
WOLCOTT H. F. (1994), Transforming qualitative data: description, analysis and interpretation, Thousand Oaks : Sage. 